Gut, 1974, 15, 130-131

\title{
The sequential deposition of crystalline material in gallstones : Evidence for changing gallbladder bile composition during the growth of some stones
}

\author{
D. JUNE SUTOR AND SUSAN E. WOOLEY \\ From the Department of Chemistry, University College, Gower Street, London
}

SUMMARY Determination of the sequential deposition of crystalline compounds in a representative collection of gallstones from 578 patients has shown that a change in composition of gallstones containing both cholesterol and calcium salt(s) has frequently occurred during growth. Generally a central stone area composed of one of these types of compound is surrounded by outer layers formed of a mixture of both, or vice versa. In a few stones, however, there is a change from one type of compound to the other occurring in successive layers. Both in stones containing cholesterol and calcium salt(s) and in those consisting entirely of calcium salt, there is often also a change in the type of calcium salt present. Such changes provide indirect evidence of changes in gallbladder bile composition during growth of stones, the origin and mechanism of which are not understood.

Although cholesterol is the crystalline compound most frequently found in gallstones, certain crystalline calcium salts are often present in varying amounts accounting for 3 to $100 \%$ of the total crystalline material of the stone (Sutor and Wooley, 1973). The calcium salts may be one or more of the following: calcium palmitate, calcium bilirubinate, the three forms of calcium carbonate-vaterite, aragonite, and calcite - and the two forms of calcium phosphate-apatite and whitlockite. Cholesterol and each calcium salt are likely to require different conditions for their precipitation from bile, and an investigation of the sequential deposition of the constituents in gallstones, particularly those that are not homogeneous in composition, provides information not only on the structure of the stones themselves but also on the composition of bile during stone nucleation and growth. Since no such detailed study has been reported, the sequential deposition of the constituents has been determined in all gallstones analysed by us and the results and their implications are summarized here.

\section{Material and Methods}

This survey includes $\mathbf{5 7 8}$ gallstones from the same number of patients, made up of representative collections from several countries but dominated

Received for publication 14 November 1973. by the one from England (212 stones). To obtain a representative collection from a particular area, the stones were collected over a period of time as patients presented for operation.

Each gallstone was cut carefully in half and small samples of material for analysis were removed from the nucleus, interior, and surface, more than one being taken from a particular area which appeared to be inhomogeneous. In the case of very small stones when it was not usually possible to sample the different areas separately, only one representative specimen was taken. The crystalline constituents present in the samples were determined by the $x$-ray powder method (Sutor and Wooley, 1971). For presentation of some results, these constituents were broadly classified as cholesterol or calcium salts, as suggested previously (Sutor and Wooley, 1973).

\section{Results}

There were 249 stones consisting entirely of cholesterol or of cholesterol plus the small white beads of calcium palmitate (Sutor, 1970), 77 composed entirely of calcium salt(s), 242 containing both cholesterol and calcium salt(s) in varying proportions, and 10 of amorphous or unidentified material. Since the different cholesterols found in gallstones are all probably laid down in vivo as cholesterol monohydrate (Sutor and Wooley, 1971; Sutor and Gaston, 1972) and the calcium palmitate 
beads appear to be incorporated in the stone rather than to grow with it, the sequential deposition in these stones is not important in showing gallstone structure and changing bile composition.

The sequential deposition of cholesterol and calcium salt(s) in stones of mixed composition is given in the table. In 76 of the 207 gallstones from

\begin{tabular}{rlll}
\hline $\begin{array}{l}\text { Number of } \\
\text { Stones }\end{array}$ & \multicolumn{2}{l}{ Composition of } & \\
\cline { 2 - 4 } & Nucleus & Interior & Surface \\
\hline 76 & Mixed & Mixed & Mixed \\
34 & Ch$^{1}$ & Mixed & Mixed \\
39 & Ch & Ch & Mixed \\
4 & $\mathrm{Ca}^{1}$ & Mixed & Mixed \\
6 & Ca & Ca & Mixed \\
5 & Mixed & Ch & Ch \\
15 & Mixed & Mixed & Ch \\
3 & Mixed & Mixed & Ca \\
8 & Ch & Mixed & Ch \\
6 & Mixed & Ch & Mixed \\
2 & Ca & Ch & Ch \\
4 & Ca & Ca & Ch \\
1 & Mixed & Ch & Ca \\
3 & Ch & Mixed & Ca \\
1 & Ca & Mixed & Ch \\
35 & Only one composite sample taken. & \\
\hline
\end{tabular}

Table Sequential deposition of constituents in 242 gallstones containing both cholesterol and calcium salt(s)

${ }^{1} \mathrm{Ch}=$ cholesterol, $\mathrm{Ca}=$ calcium salt(s), mixed $=$ cholesterol + calcium salt(s).

which several samples could be analysed, both types of compound were present as an intimate mixture throughout the stones. In the remaining 131, however, a change in composition has occurred during growth. A nucleus or nucleus plus interior, usually of cholesterol but sometimes of calcium salt(s), is surrounded by an area consisting of both types of compound, or stones with nuclei or nuclei plus interiors of both cholesterol and calcium salt(s) have only one of these compounds on the outside. Of particular interest are the gallstones in which there is an abrupt change in composition from cholesterol to calcium salt(s) or vice versa in successive stone layers or with a band of mixed composition occurring between areas of cholesterol and calcium salt(s).

Because of the number of calcium salts and their different possible combinations throughout the stones, the sequential deposition of these constituents could not be studied in detail. It became apparent, however, that the presence of a particular calcium salt in the nucleus did not imply its occurrence throughout the stone and changes in the number and type of calcium salts precipitated could occur as well as changes in the crystalline form of calcium carbonate and calcium phosphate.

\section{Discussion}

A study of the sequential deposition of compounds in gallstones has shown that the composition has often changed during growth. Since nucleation and growth of all crystalline material are governed by the same laws, a change in gallstone composition provides indirect evidence of a change in gallbladder bile composition. For example, a gallstone that has a nucleus and interior of cholesterol and a surface layer of calcium carbonate must have nucleated and partly grown in bile that was supersaturated with cholesterol, but after a time the bile in contact with the stone became undersaturated or just saturated with respect to cholesterol and supersaturated with calcium carbonate.

Extensive changes in bile composition during the growth of some gallstones, as suggested by this analysis, might result from an alteration in the composition of hepatic bile or from a change occurring within the gallbladder. Loss of gallbladder function might in some cases be responsible for the cessation of calcium salt deposition since supersaturation of the bile with respect to the calcium salt might have resulted from the concentrating action of the gallbladder. Loss of gallbladder function would not, however, explain the appearance of calcium salt(s) in a stone or the appearance or disappearance of cholesterol.

Studies of the composition of bile during growth of stones are necessary to elucidate the extent, origin, and mechanism of these changes which further illustrate the complexity of gallstone formation.

We thank the Medical Research Council for financial support.

\section{References}

Sutor, D. J. (1970). Calcium palmitate and a-palmitic acid in gallstones. Gut, 11, 618-619.

Sutor, D. J., and Gaston, P. J. (1972). Anhydrous cholesterol: a new crystalline form in gallstones. Gut, 13, 64-65.

Sutor, D. J., and Wooley, S. E. (1971). A statistical survey of the composition of gallstones in eight countries. Gut, 12, 55-64.

Sutor, D. J., and Wooley, S. E. (1973). The nature and incidence of gallstones containing calcium. Gut, 14, 215-220. 